\title{
Plasma Theory of Two Synchrotron Knots' formation Discovered in the Crab Nebula
}

\author{
David Shapakidze and George Machabeli \\ Georgian Academy of Sciences, Abastumani Astrophysical Observatory, \\ Al. Kazbegi ave. 2a, 380060, Tbilisi, Georgia
}

\begin{abstract}
The plasma mechanism of synchrotron knots' formation discovered in the Crab Nebula at the distances $0^{\prime \prime} .65\left(10^{16} \mathrm{~cm}\right)$ and $3^{\prime \prime} .8$ $\left(6 \times 10^{16} \mathrm{~cm}\right)$ from the Crab pulsar is presented. The mechanism is based on exitation of cyclotron and Cherenkov-drift instabilities in the relativistic electron-positron plasma of the nebula. The higher luminosity of the knots is supposed due to the orientation of the direction of motion of the synchrotron radiation sources (Larmor circles) relative to the observer.
\end{abstract}

\section{Introduction}

The aim of the work proposed is theoretical explanation of two bright synchrotron knots appeared on the images of the Crab synchrotron Nebula obtained with the Wide Field and Planetary Camera 2 (WFPC2) on the board of the Hubble Space Telescope by Hester et al. (1995). The knots are located at $0^{\prime \prime} .65\left(2 \times 10^{16} \mathrm{~cm}\right)$ and $3^{\prime \prime} .8\left(10^{17} \mathrm{~cm}\right)$ from the pulsar and radiate optical synchrotron emission. We suggest the constant, pulsar rotation independent, structure of magnetic field frozen with relativistic electron positron plasma. We assume that relativistic $e^{-} e^{+}$plasma moves along these magnetic field lines. However the motion of particles is force-free - magnetic field lines are reconstructing under the motion of particles in the force-free manner. At large enough distances $\left(R \geq 10^{\mathbf{1 4}} \mathrm{cm}\right)$ (Machabeli, Mchedlishvili and Shapakidze, 1998) this reconstruction gives the constant, pulsar rotation independent structure of magnetic field.

\section{The Mechanism of Knot's Formation}

Plasma is composed from the following parts near the star surface: the bulk of the electron-positron plasma with an average Lorentz factor $\gamma_{p} \approx 3$ and the number density $n_{p}^{0} \sim 10^{16} \mathrm{~cm}^{-3}$ (Machabeli and Usov 1989); a tail of an energetic electrons an positrons with $\gamma_{t}=10^{4} \div 10^{5}$ and $n_{t}^{0} \sim 10^{13} \div 10^{14} \mathrm{~cm}^{-3}$ and a primary beam of ions $\left(n_{b_{i}}^{0} \sim 10^{8} \mathrm{~cm}^{-3}\right)$ or electrons $\left(n_{b_{e}}^{0} \sim 10^{11} \mathrm{~cm}^{-3}\right)$ with characteristic Lorentz factors $\gamma \sim 10^{6} \div 10^{7}$ (Arons 1981). It is assumed that such distribution function of particles does not change up to the distances $10^{16} \div 10^{17} \mathrm{~cm}$.

According to the plasma linear theory, in such a plasma, purely transversal $t$ and longitudinal-transversal $l t$ waves should be generated at the Cherenkov- 
drift and at anomalous Doppler-effect resonances at the distances the knots have been disovered. The excited waves are acting on the distribution function of the particles and this interaction is described by the plasma quasilinear theory. The transverse momentums of the particles relative to the magnetic field lines are appeared. It means: pitch-angles appeared to the ion or electron beam particles due to Cheren kov-drift instability and to the tail particles due to cyclotron instability and they radiate synchrotron radiation. The frequency of the synchrotron radiation in the frame of larmor circle may be calculated as $\nu_{0} \simeq 0.07(e B / m c) \gamma^{2} \psi_{\text {eff }}$ (here $\psi_{e f f}$ is the effective pitch-angle). The maximal radiating power per frequency unit is $P_{\nu_{0}}=(1.6 / 2 \pi)\left(e^{2} / c\right) \omega_{B}$. On Earth, the frequency and the radiating power are measured according to the well-known transformation formulae: $\nu=\nu_{0} \sqrt{1-V^{2} / c^{2}} /(1-V \cos \alpha / c)$ and $P_{\nu}=P_{\nu_{0}} /(1-V \cos \alpha / c)$ (Landau and Lifshitz 1975), where $\alpha$ is the angle between the direction of radiation (the observer's axis in our case) and the magnetic field.

The linear velocities of larmour circles of the radiated particles are directed along the tangent of the curve magnetic field lines. Then the observed frequency $\nu$ should not differ from $\nu_{0}$ and the radiative power $P_{\nu}$ should $\gamma$ times greater than $P_{0}$ if we assume that the angle $\alpha$ between the line of sight and the direction of the tangent of the magnetic field line in the point of knots' location fulfilles the condition $\cos \alpha=1-\left(1 / \gamma_{\text {res }}\right)$. It is easy to find the formula for observed flux densities $f_{\nu}$ using the formula for $P_{\nu_{0}}$ :

$$
f_{\nu}=\frac{1.6}{2 \pi} \frac{e^{3} B_{L C}}{c^{2}} n_{0}^{r} V R_{0} \frac{1}{4 \pi L^{2}} \frac{\psi_{\text {eff }} \gamma_{r e s}}{m R^{3}},
$$

where $L$ is the distance from the Earth to the Nebula equal $2000 \mathrm{kpc}$ and $V$ is the approximate volume of the knots which is $8.4 \times 10^{47} \mathrm{~cm}^{3}$ for the knot1 and $4 \times 10^{47} \mathrm{~cm}^{3}$ for the knot2 according to Hester et al. 1995 . Estimating the pitch angles we find that the electron-beam particles are resposible via. Cherenkov-drift instability for the knot 1 formation at the distance from the pulsar $0^{\prime \prime} 65$ and tail particles are responsibe via cyclotron instability for the knot2 formation at the distance from the pulsar $3^{\prime \prime} .8$.

For the electron-beam particles we have: $\gamma \sim 10^{7} ; \quad \psi_{\text {eff }} \sim 10^{-3} ; \quad \nu \sim$ $10^{14} s^{-1} ; f_{\nu} \sim 10^{-28} \mathrm{erg} \mathrm{cm}^{-2} \mathrm{~s}^{-1} \mathrm{~Hz}^{-1}$; For the tail particles we have: $\gamma \sim$ $10^{6} ; \psi_{e f f} \sim 10^{-1} ; \nu \sim 10^{14} \mathrm{~s}^{-1} ; f_{\nu} \sim 10^{-29} \mathrm{erg} \mathrm{cm}^{-2} \mathrm{~s}^{-1} \mathrm{~Hz}^{-1}$.

The values $f_{\nu}$ and frequencies $\nu$ are in good correlation with observational data obtained by Hubble Space Telescope (Hester et al. 1995)

\section{References}

Arons J. 1981, in Proc. Varenna Summer School and Workshop on Plasma Astrophysics, ESA, 273.

Hester, J. J. et.al 1995, ApJ, 448, 240.

Landau, L. D., \& Lifshitz, E. M., The Classical Theory of Fields (Pergamon Press, Oxford 1975).

Machabeli, G. Z., \& Usov, V. V. 1989, Sov. Astron. Lett., 15, 393.

Machabeli, G., Mchedlishvili, G., \& Shapakidze, D. 1998, Astron. Space Sci., submitted. 